\title{
Long-lived circulating currents in strongly correlated nanorings
}

\author{
B. M. Schoenauer, ${ }^{1}$ N. M. Gergs, ${ }^{1}$ P. Schmitteckert, ${ }^{2,3}$ F. Evers, ${ }^{4}$ and D. Schuricht ${ }^{1}$ \\ ${ }^{1}$ Institute for Theoretical Physics, Center for Extreme Matter and Emergent Phenomena, Utrecht University, \\ Princetonplein 5, 3584 CE Utrecht, Netherlands \\ ${ }^{2}$ Institute for Theoretical Physics and Astrophysics, Julius-Maximilians University of Würzburg, Am Hubland, 97074 Würzburg, Germany \\ ${ }^{3}$ HQS Quantum Simulations GmbH, 76131 Karlsruhe, Germany \\ ${ }^{4}$ Institute of Theoretical Physics, University of Regensburg, 93040 Regensburg, Germany
}

(Received 23 February 2019; published 17 September 2019)

\begin{abstract}
We study the time-evolving currents flowing in an interacting ring-shaped nanostructure after a bias voltage has been switched on. The source-to-drain current exhibits the expected relaxation towards its quasistatic equilibrium value at a rate $\Gamma_{0}$ reflecting the lead-induced broadening of the ring states. In contrast, the current circulating within the ring decays with a different rate $\Gamma$, which is a rapidly decaying function of the interaction strength and thus can take values orders of magnitude below $\Gamma_{0}$. This implies the existence of a regime in which the nanostructure is far from equilibrium even though the transmitted current is already stationary. We discuss experimental setups to observe the long-lived ring transients.
\end{abstract}

DOI: 10.1103/PhysRevResearch.1.022006

Introduction. Isolated quantum systems, such as small molecules, feature a discrete set of energy levels. When brought into contact with two electrodes, a nanojunction can form and a current begins to flow. At weak coupling, the associated level broadening $\Gamma_{0}$ is still small as compared to the typical energy spacing $\Delta E$ of the isolated system. One might perhaps suspect that these energies by themselves set the only relevant timescales. However, in fact a prominent exception is known, the Kondo phenomenon [1], which occurs in a situation where $\Delta E$ is dominated by strong on-site repulsion between the charge carriers. This suppresses charge fluctuations but allows for spin fluctuations, leading to an emergent energy scale, the Kondo temperature $T_{\mathrm{K}}$, which is parametrically small compared to the native scales $\Gamma_{0}$ and $\Delta E$.

In this work we report another example of an emergent energy scale $\Gamma$, which manifests in the relaxation of circulating currents in mesoscopic nanostructures. Like the Kondo temperature, this scale is a many-body phenomenon, originating from interactions between particles on the nanostructure. However, the manifestation of the relaxation rate $\Gamma$ requires the nanostructure to be brought out of equilibrium.

A sketch of a minimal model system that exhibits the scale $\Gamma$ is displayed in Fig. 1. Originally, similar ring-shaped devices served as toy models to study the interplay of interaction and interference [2,3] and to explain quantum-interference effects in transport through functionalized graphene ribbons [4]. The ring geometry supports stationary circulating (orbital) currents that can exceed the source-drain (transport) current

Published by the American Physical Society under the terms of the Creative Commons Attribution 4.0 International license. Further distribution of this work must maintain attribution to the author(s) and the published article's title, journal citation, and DOI. by orders of magnitude at Fermi energies situated close to a Fano resonance.

Strong circulating currents in ring-shaped devices (Fig. 1) generically arise as transients after a voltage quench. They then carry an oscillating amplitude with a frequency resembling the lowest-lying excitation gap of the nanostructure. We report here results from time-dependent density matrix renormalization group (TDDMRG) [5-8] simulations showing that in situations where the interaction $U$ is the dominating native scale of the nanoring, these oscillations can be very pronounced and very long lived. They exhibit a lifetime $\Gamma^{-1}$ that exceeds the transients in transport currents $\Gamma_{0}^{-1}$ by orders of magnitude if the repulsive interaction $U$ becomes strong. The computational finding is complemented with perturbative arguments that explain this effect and clarify the relevant physical processes. In essence, the strong suppression of $\Gamma$ originates in a large energy gap between the two low-lying states and the rest of the spectrum on the nanoring (see Fig. 3), with ring current connecting the low-lying states. Possible experimental signatures of the effect proposed here are discussed. We note that in contrast to previously discussed [9-11] persistent ring currents driven by magnetic fields, the oscillating ring current we observe is a pure nonequilibrium effect.

Nanostructure. The model associated with Fig. 1 is represented by the Hamiltonian $H=H_{\mathrm{r}}+H_{1}+H_{\mathrm{c}}$ describing the ring, the leads, and their mutual coupling, respectively. The ring Hamiltonian is given by

$$
\begin{aligned}
H_{\mathrm{r}}= & -J \sum_{\langle i, j\rangle}\left(d_{i}^{\dagger} d_{j}+d_{j}^{\dagger} d_{i}\right) \\
& +U \sum_{\langle i, j\rangle}\left(n_{i} n_{j}-\frac{n_{i}+n_{j}}{2}\right)+\varepsilon_{T} n_{2},
\end{aligned}
$$

with operators $d_{j}^{\dagger}$ and $d_{j}$ creating and annihilating spinless fermions at site $j$ and $n_{j}=d_{j}^{\dagger} d_{j}$ defining the corresponding 


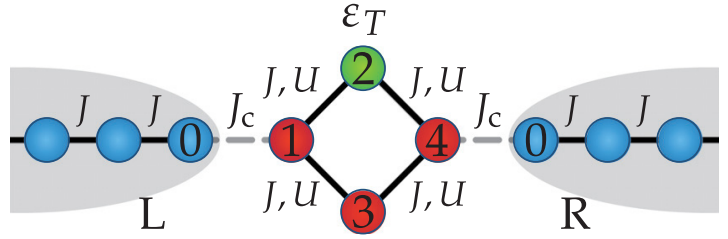

FIG. 1. Schematic representation of the nanostructure. The ring (green and red circles) is coupled by $J_{\mathrm{c}}$ to left and right leads (blue circles). Spinless fermions can hop within the ring and leads with amplitude $J$, the top site (site 2) on the ring is subject to the potential $\varepsilon_{T}$, and inside the ring a nearest-neighbor interaction $U$ is present.

density. The first term describes hopping of the fermions between nearest neighbors, while the second represents the repulsive nearest-neighbor interaction. The last term is an external potential at the top site which breaks the symmetry between the upper and lower paths through the ring. The lead Hamiltonian reads

$$
H_{1}=-J \sum_{\alpha=\mathrm{L}, \mathrm{R}} \sum_{n \geqslant 0}\left(c_{\alpha, n+1}^{\dagger} c_{\alpha, n}+c_{\alpha, n}^{\dagger} c_{\alpha, n+1}\right),
$$

where $c_{n, \alpha}^{\dagger}$ and $c_{n, \alpha}$ create and annihilate a spinless fermion at site $n$ in the lead $\alpha=\mathrm{L}, \mathrm{R}$. For simplicity, we assume the hopping parameter $J$ in the ring and lead to be equal. Finally, the coupling between both subsystems is facilitated by

$$
H_{\mathrm{c}}=-J_{\mathrm{c}}\left(d_{1}^{\dagger} c_{\mathrm{L}, 0}+c_{\mathrm{L}, 0}^{\dagger} d_{1}+d_{4}^{\dagger} c_{\mathrm{R}, 0}+c_{\mathrm{R}, 0}^{\dagger} d_{4}\right),
$$

coupling the outer sites on the ring to the leads.

In the following we analyze the nonequilibrium currents in the nanostructure by three different methods: (i) TDDMRG simulations, (ii) a reduced density-operator transport theory (RDTT) $[12,13]$, and (iii) mapping to an effective two-state nanostructure [14].

TDDMRG simulations. First, we study the time evolution after a voltage quench using the TDDMRG algorithm [15-21]. Specifically, we use the time evolution scheme outlined in Refs. [8,21,22] performing the evaluation of the time evolution via matrix exponentials within the framework of Krylov spaces. At times $t<0$ the system is prepared in the ground state of the model with an additional charge excess induced by a stationary gating with $V / 2\left(\sum_{i} n_{\mathrm{L}, i}-\sum_{i} n_{\mathrm{R}, i}\right)$. At $t=0$ the gate is switched off, so the electrodes begin to discharge and currents start to flow through the system. We simulate the time evolution with finite leads which are long enough to be able to study the transient regime all the way into the quasistationary nonequilibrium limit. Finitesize effects will interfere only at times exceeding the recurrence time $\tau_{\mathrm{rec}}=L / 2 v_{\mathrm{F}}$, at which the electrons reach the boundary of the leads. (For details of the quenching protocol see Ref. [21].) Here $L$ denotes the total number of sites, i.e., the length of the leads is given by $(L-4) / 2 \approx L / 2$, and $v_{\mathrm{F}}=2 J$ is the Fermi velocity of the lead electrons.

During the time evolution we determine the expectation values of the local currents $I_{\mathrm{t}} \propto \operatorname{Im}\left(c_{i}^{\dagger} c_{i-1}\right)$ and $I_{\mathrm{r}} \propto \operatorname{Im}\left(d_{k}^{\dagger} d_{l}\right)$ flowing in the leads and the impurity, respectively, where $l$ and $k$ are neighboring sites. The local current densities after quenching are displayed in Fig. 2. The transport (transmitted) current $I_{\mathrm{t}}$ initially fluctuates in response to the quench for times $\Gamma_{0} t \leqslant 3$, where we use $\Gamma_{0}=2 \pi \rho_{0} J_{\mathrm{c}}^{2}$ with the density

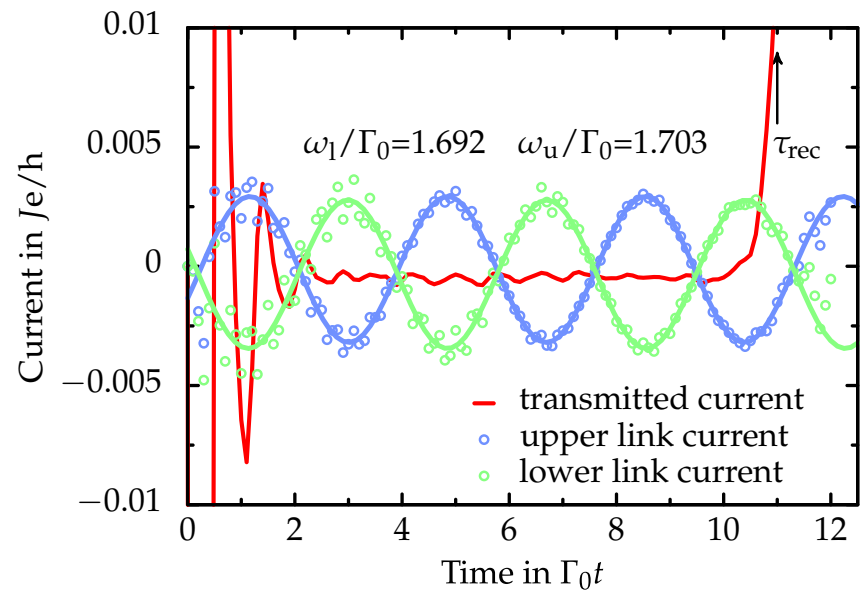

FIG. 2. Time evolution of the transmitted and ring currents $\left\langle I_{\mathrm{t}}\right\rangle(t)$ and $\left\langle I_{\mathrm{r}}\right\rangle(t)$ evaluated using TDDMRG on the links $1 \rightarrow 2$ and $1 \rightarrow$ 3 in Fig. 1 . The recurrence time $\tau_{\text {rec }}=L / 2 v_{\mathrm{F}} \simeq 44$ is indicated by the black arrow. While the transmitted current quickly relaxes to a stationary value, the ring currents show persistent oscillations with frequencies $\omega_{1, \mathrm{u}}$ over the accessible times. The simulation parameters are $L=96, U=4 J, \varepsilon_{T}=J / 2, J_{\mathrm{c}}=J / 2$, and $\mathrm{e} V=0.4 J$.

of states in the leads $\rho_{0}=1 / 2 \pi J$ as our time unit. After this transient, the transmitted current appears to have reached a largely time-independent steady state in line with predictions from nonequilibrium Green's function formalism [23,24].

In contrast, for the local currents in the ring $I_{\mathrm{r}}$ we observe a drastically different behavior. Although some transient features decay quickly, the ring currents oscillate with a distinct frequency $\omega$ for long times. In fact, for sufficiently strong Coulomb repulsions $U$ we do not observe a significant reduction of the oscillation amplitude within the observation times accessible to our simulations. Qualitatively similar results were obtained for a ring structure with eight sites [22].

The frequency of the oscillations can be understood based on the spectrum [22] of the uncoupled ring $H_{\mathrm{r}}$ shown in Fig. 3. We find that the frequency $\omega$ extracted from the TDDMRG simulations matches the energy gap between the

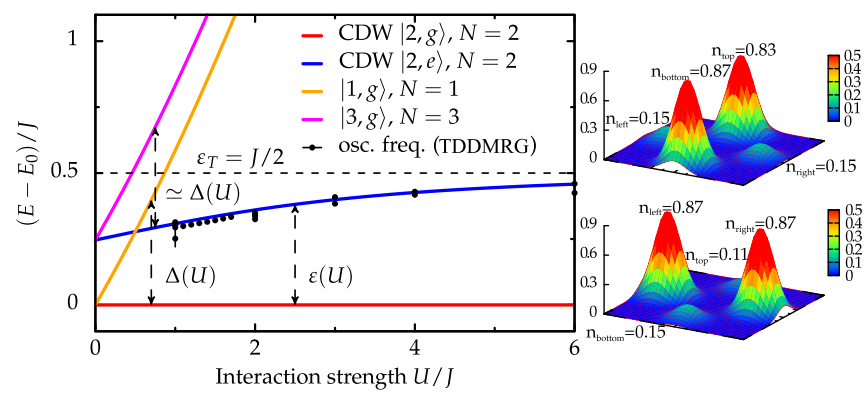

FIG. 3. Spectrum of the uncoupled ring $H_{\mathrm{r}}$ relative to the groundstate energy $E_{0}$. The ground state $|2, g\rangle$ is a CDW state with $N=2$ particles; for $U>J$ the first excited state $|2, e\rangle$ is also a CDW state with two particles. The corresponding particle densities are shown for $U=2 J$. The observed oscillation frequencies of the ring currents match the energy difference $\varepsilon(U)$ between these two states. The higher excited states are obtained by adding or removing particles, with $\Delta(U)$ denoting the corresponding energies. 


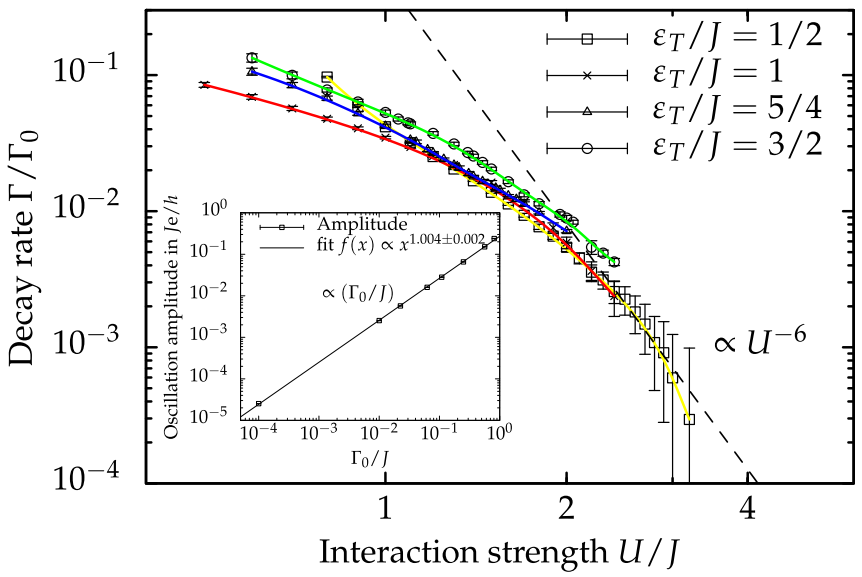

FIG. 4. Decay rate $\Gamma$ of the ring current extracted from TDDMRG simulations. For $U / \varepsilon_{T} \simeq 1$ the decay rate appears to be exponentially suppressed in $U$. For $U \gg \varepsilon_{T}$ the decay is consistent with $\Gamma \sim U^{-6}$ predicted using an effective two-level system (5), as is indicated by the dashed line. All other parameters are as in Fig. 2. The inset shows the dependence of the amplitude of the ring current on the coupling $\Gamma_{0}$ to the leads.

two

lowest-lying states on the ring. These two states can be identified as charge-density wave (CDW) states with $N=2$ particles on the ring, one being the ground state $|2, g\rangle$ and the other the first excited state $|2, e\rangle$. Thus we confirm that the ring current originates from the mixing of these two states by the time evolution, which is driven by the coupling of the ring to the leads as exemplified by the proportionality of the ring current to the coupling $\Gamma_{0}$ shown in the inset of Fig. 4.

The decay rate $\Gamma$ of the ring currents is rapidly decreasing with the interaction strength $U$ (see Fig. 4), exhibiting a wide regime with $\Gamma \ll \Gamma_{0}$. To understand the origin of this regime, we proceed with the RDTT analysis.
RDTT analysis. The RDTT [12,13] method aims at determining the time evolution of the reduced density matrix of the nanostructure $\rho_{\mathrm{ns}}(t)=\operatorname{tr}_{1} \rho(t)$, where the trace is taken over the lead degrees of freedom in the density matrix $\rho(t)$ of the full system. The time evolution of $\rho_{\mathrm{ns}}(t)$ can be cast in the form $\dot{\rho}_{\mathrm{ns}}(t)=-i L_{\mathrm{ns}} \rho_{\mathrm{ns}}(t)$, with the effective Liouvillian $L_{\mathrm{ns}}$ governing the relaxation of the nanostructure. Since the ring current originates from the mixing of the two CDW states $|2, g\rangle$ and $|2, e\rangle$, its decay is related to the decay of the offdiagonal elements $\rho_{g e}$ and $\rho_{e g}$ of $\rho_{\mathrm{ns}}$. We have determined the corresponding decay rate from the Liouvillian $L_{\mathrm{ns}}$ calculated [22] to first order in the bare coupling rate $\Gamma_{0}$, with the perturbative regime set by $\Gamma_{0} \ll T$ with the temperature $T$.

The obtained results for the decay rate $\Gamma$ of the ring current are shown in Fig. 5(a). The results are qualitatively similar to the ones obtained via TDDMRG shown in Fig. 4 in the sense that the rate is strongly suppressed at large $U$. The quantitative differences between the RDTT and TDDMRG results reflect the fact that both methods operate in different parameter regimes.

Furthermore, the RDTT allows us to identify [22] the relaxation processes contributing to the decay rate, which are visualized in Fig. 5(b). The dominant processes are shown in sketches (1) and (2), which involve the tunneling of a particle off or onto the ring, while the subleading processes are shown in sketches (3) and (4). All processes are constraint by energetics: Sketches (1) and (2) only contribute in the regions (i) and (ii) in Fig. 5(a), (3) contributes only in regions (i) and (iii), and (4) is relevant in regions (i)-(iv). We stress that in region $(v)$ no relaxation processes to order $\Gamma_{0}$ exist. Thus, at sufficiently large interaction strengths $U$, the rate $\Gamma$ essentially drops to zero (to order $\Gamma_{0}^{2}$ ), explaining the very slow decay of the ring current.

Schrieffer-Wolff transformation. Finally, we focus on the regime of strong interactions $U / \max \left(\varepsilon_{T}, J\right) \rightarrow \infty$, where we can derive the analytic dependence $\Gamma \sim U^{-6}$ consistent with (a)

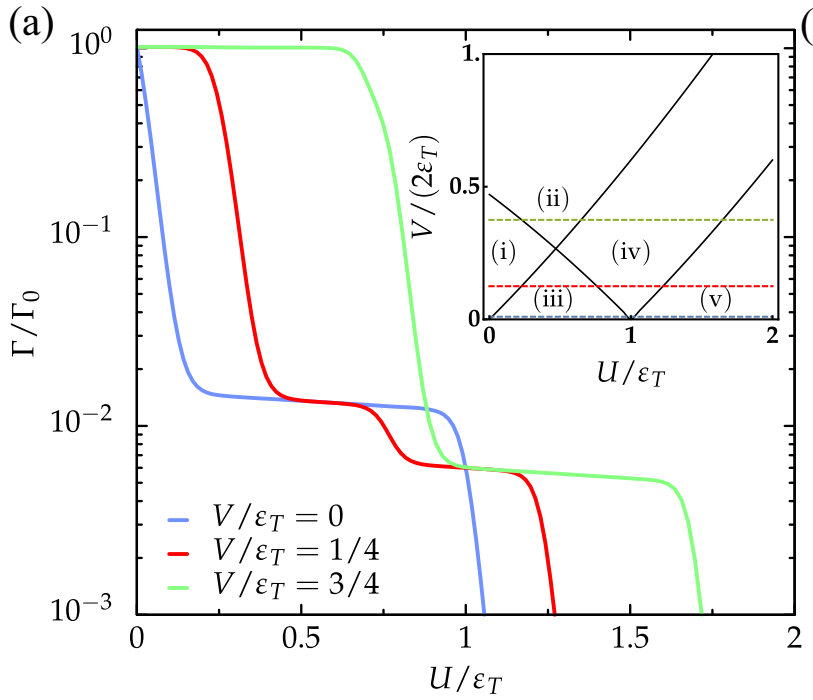

(b)

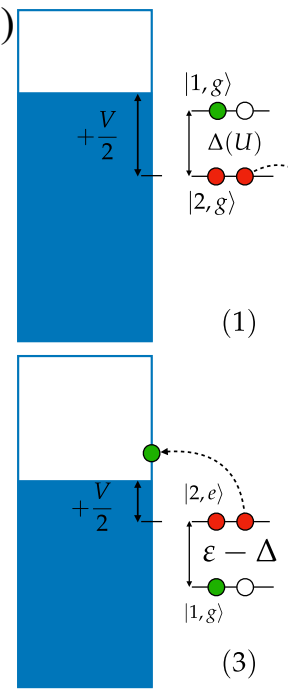

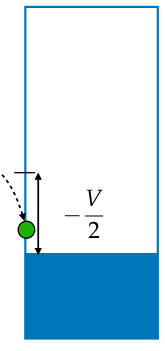
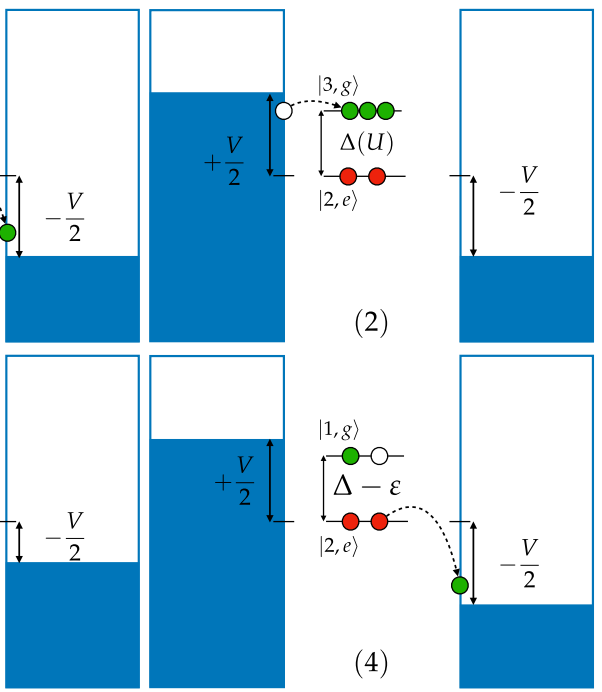

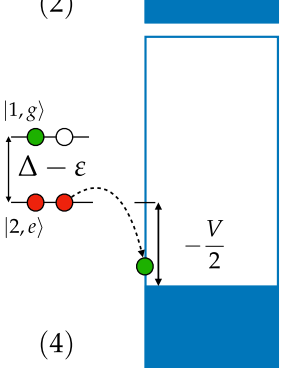

FIG. 5. (a) Decay rate $\Gamma$ obtained from RDTT for the temperature $T=10 \Gamma_{0}$. The inset shows that in the $U-V$ parameter space we identify five distinct regions labeled (i) $-(\mathrm{v})$, in which $\Gamma$ takes very different values. The dashed lines indicate cuts shown in the main figure. (b) Relaxation processes contributing to the rate $\Gamma$, which result in the distinct regions (i)-(v). Red and green circles represent initial and final configurations, respectively, $\Delta=\Delta(U)$ denotes the energy required to add or remove a particle (see Fig. 3), and $\varepsilon \approx \varepsilon_{T}$ is the energy gap between the two CDW states. 
our computational results (Fig. 4). As can be seen from the spectrum of the bare ring (Fig. 3), in this limit the two CDW states $|2, g\rangle$ and $|2, e\rangle$ will be well separated by an energy splitting $\Delta(U) \sim U$ from the higher excited states. It is thus instructive to construct an effective two-level system containing only these states, where the couplings to the higher excited states are treated using a Schrieffer-Wolff transformation [14] to fourth order in the couplings $J$ and $J_{\mathrm{c}}$. Going to this order in the expansion is necessary since all off-diagonal matrix elements exactly cancel in second order due to the mirror symmetry of the isolated ring structure [22].

The resulting two-level system can be written in the form of an electronic Kondo model, with the localized spin identified with the CDW states as $|\downarrow\rangle=|2, g\rangle$ and $|\uparrow\rangle=|2, e\rangle$ and the corresponding spin operator denoted by $\vec{S}$. An effective reservoir electronic degree of freedom can be formed via $c_{\text {res, } \uparrow \downarrow}=\left(c_{\mathrm{L}} \pm c_{\mathrm{R}}\right) / \sqrt{2}$ from the leads (2) of the original model; the effective spin operator formed from the first sites ( $n=0)$ is denoted by $\vec{S}_{\text {res }}$. With this notation the effective model reads [22]

$$
\begin{aligned}
H_{\mathrm{SW}}= & \sum_{k, \sigma} \epsilon_{k} c_{\mathrm{res}, k \sigma}^{\dagger} c_{\mathrm{res}, k \sigma}+h S^{z}+\tilde{h} S_{\mathrm{res}}^{z} \\
& +J_{\perp}\left(S^{x} S_{\mathrm{res}}^{x}+S^{y} S_{\mathrm{res}}^{y}\right)+J_{z} S^{z} S_{\mathrm{res}}^{z},
\end{aligned}
$$

where the first term is the energy of the electronic reservoir, the second and third are effective magnetic fields $h \approx \varepsilon_{T}$ and $\tilde{h}=O\left(U^{-4}\right) \ll h$ acting on the two-level system and spin of the electron reservoir, and the fourth and fifth terms represent a Kondo coupling between the two, with the coupling being strongly anisotropic with $J_{z} \simeq 10 J^{2} J_{\mathrm{c}}^{2} / U^{3}$ and $J_{\perp}=O\left(U^{-5}\right)$, and thus $\left|J_{\perp}\right| \ll\left|J_{z}\right| \ll J_{\mathrm{c}}, J$.

Due to the formation of the effective reservoir electron spin from the leads L, R, the bias voltage $V$ enters the effective Kondo model in the form of a transverse field in the reservoir, i.e., as $V / 2 \sum_{k \sigma \sigma^{\prime}} c_{\text {res }, k \sigma} \tau_{\sigma \sigma^{\prime}}^{x} c_{\text {res }, k \sigma^{\prime}}$, with $\tau^{x}$ the $x$ component of the Pauli matrices. Finally, the ring current corresponds to oscillations between the two CDW states and thus is related to the localized spin via $I_{\mathrm{r}} \sim S^{y}$. Performing a suitable spin rotation in the electronic reservoir, we calculated [22] the corresponding relaxation rate using standard perturbation theory in the Kondo system [12,25,26], with the result

$$
\Gamma=\frac{\pi \rho_{0}^{2} J_{\perp}^{2}}{16}\left(\left|\varepsilon_{T}+V\right|+\left|\varepsilon_{T}-V\right|+2\left|\varepsilon_{T}\right|\right)+\frac{\pi \rho_{0}^{2} J_{z}^{2}}{8} V .
$$

We stress that in the considered regime of strong interactions this rate is vanishingly small, $\Gamma \sim \rho_{0}^{2} J_{z}^{2} V \sim \rho_{0}^{2} J^{4} J_{\mathrm{c}}^{4} V / U^{6}$, in accordance with our finding of long-lived oscillations in the ring current. In particular, the predicted behavior $\Gamma \sim U^{-6}$ is consistent with our TDDMRG simulations shown in Fig. 4. We note that the result (5) is applicable deep in region (v) of Fig. 5(a), where we found that processes of order $\Gamma_{0}$ vanish. Furthermore, the effective model (4) will show the Kondo effect; however, the relevant energy scale $T_{\mathrm{K}}$ will be much smaller than the energy scales we consider here, in particular $T_{\mathrm{K}} \ll \varepsilon_{T}$. Thus the equilibrium Kondo effect is not observable in our setup.

Finally, we note that nanostructures with two energetically well separated low-lying states can generically be approximated by an effective Kondo model using a SchriefferWolff transformation. In the absence of the above-mentioned mirror symmetry, the exchange couplings will be of the or$\operatorname{der} J_{z}, J_{\perp} \sim J_{\mathrm{c}}^{2} / U \ll J_{\mathrm{c}}$, resulting in a relaxation rate $\Gamma \sim$ $\rho_{0}^{2} J_{z}^{2} V \sim U^{-2} \ll \Gamma_{0}$. Thus ring currents that couple to these low-lying states are still expected to decay very slowly.

Experimental verification. We see a possible experimental realization of the ring-shaped model system (Fig. 1) in molecules such as porphyrins or phthalocyanines. Singlemolecule conductance measurements have indeed been performed on these systems [27-29], so the possibility for bias ramping has also been demonstrated already. As an observable indicating the slow decay of the ring currents, we propose to measure the photons that are emitted when these currents decay via coupling to the radiation field. In this context we note that single-molecule electroluminescence measurements have been performed [30,31] already and thus are indeed experimentally feasible. An alternative realization of our ringshaped model may be provided by quantum dot arrays [32], which in particular offer a high level of control of the couplings and allow one to enter the regime of strong interactions essential for the long-lived ring currents.

Conclusion. We have studied the relaxation of transport processes in an interacting ring-shaped nanostructure. Owing to a mirror symmetry of the Hamiltonian, the system supports oscillating ring currents long after the transmitted current has died out, with the ratio $\Gamma / \Gamma_{0}$ of the respective relaxation rates being strongly suppressed by the interactions. Our work provides a striking example for an untypical situation in thermodynamic relaxation processes: Two observable currents approach their equilibrium values on timescales that are parametrically separated with rates differing by orders of magnitude. In addition, our system provides insight into the field of quantum devices as we have shown that internal oscillations can be longer lived than observed in currents through the system. While we have focused on a ring-shaped nanostructure, the appearance of the suppressed relaxation rate $\Gamma$ is generally expected in systems that can be effectively described by a two-level model with the ring current connecting the low-lying states.

Acknowledgments. We thank Theo Costi, Mikhail Pletyukhov, and Peter Wölfle for useful discussions. This work is part of the D-ITP consortium, a program of the Netherlands Organisation for Scientific Research that is funded by the Dutch Ministry of Education, Culture and Science. B.M.S. and P.S. acknowledge the HPC project QWHISTLE at the Steinbuch Centre of Computing at Karlsruhe Institute of Technology. P.S. was supported by ERC-StG-ThomaleTOPOLECTRICS-336012. F.E. thanks the DFG for support under Grants No. EV30/08-1 and No. SFB 1277, Project No. A03. B.M.S. and D.S. were supported by the Netherlands Organisation for Scientific Research under Grant No. FOM 14PR3168. 
[1] A. C. Hewson, The Kondo Problem to Heavy Fermions (Cambridge University Press, Cambridge, 1993).

[2] D. Bohr and P. Schmitteckert, The dark side of benzene: Interference vs. interaction, Ann. Phys. (Berlin) 524, 199 (2012).

[3] P. Schmitteckert, The dark side of DFT based transport calculations, Phys. Chem. Chem. Phys. 15, 15845 (2013).

[4] M. Walz, J. Wilhelm, and F. Evers, Current Patterns and Orbital Magnetism in Mesoscopic dc Transport, Phys. Rev. Lett. 113, 136602 (2014).

[5] G. Vidal, Efficient Simulation of One-Dimensional Quantum Many-Body Systems, Phys. Rev. Lett. 93, 040502 (2004).

[6] S. R. White and A. E. Feiguin, Real-Time Evolution using the Density Matrix Renormalization Group, Phys. Rev. Lett. 93, 076401 (2004).

[7] A. J. Daley, C. Kollath, U. Schollwöck, and G. Vidal, Timedependent density-matrix renormalization-group using adaptive effective Hilbert spaces, J. Stat. Mech. (2004) P04005.

[8] P. Schmitteckert, Nonequilibrium electron transport using the density matrix renormalization group method, Phys. Rev. B 70, 121302(R) (2004).

[9] V. Meden and U. Schollwöck, Conductance of interacting nanowires, Phys. Rev. B 67, 193303 (2003).

[10] R. A. Molina, D. Weinmann, R. A. Jalabert, G.-L. Ingold, and J.-L. Pichard, Conductance through a one-dimensional correlated system: Relation to persistent currents and the role of the contacts, Phys. Rev. B 67, 235306 (2003).

[11] T. Rejec and A. Ramšak, Formulas for zero-temperature conductance through a region with interaction, Phys. Rev. B 68, 035342 (2003).

[12] H. Schoeller, A perturbative nonequilibrium renormalization group method for dissipative quantum mechanics, Eur. Phys. J. Spec. Top. 168, 179 (2009).

[13] R. B. Saptsov and M. R. Wegewijs, Fermionic superoperators for zero-temperature nonlinear transport: Real-time perturbation theory and renormalization group for Anderson quantum dots, Phys. Rev. B 86, 235432 (2012).

[14] S. Bravyi, D. P. DiVincenzo, and D. Loss, Schrieffer-Wolff transformation for quantum many-body systems, Ann. Phys. (NY) 326, 2793 (2011).

[15] D. Bohr and P. Schmitteckert, Strong enhancement of transport by interaction on contact links, Phys. Rev. B 75, 241103(R) (2007).

[16] E. Boulat, H. Saleur, and P. Schmitteckert, Twofold Advance in the Theoretical Understanding of Far-from-Equilibrium Properties of Interacting Nanostructures, Phys. Rev. Lett. 101, 140601 (2008).

[17] S. Kirino, T. Fujii, J. Zhao, and K. Ueda, Time-dependent DMRG study on quantum dot under a finite bias voltage, J. Phys. Soc. Jpn. 77, 084704 (2008).

[18] L. G. G. V. Dias da Silva, F. Heidrich-Meisner, A. E. Feiguin, C. A. Büsser, G. B. Martins, E. V. Anda, and E. Dagotto, Transport properties and Kondo correlations in nanostructures: Time-dependent DMRG method applied to quantum dots coupled to Wilson chains, Phys. Rev. B 78, 195317 (2008).
[19] F. Heidrich-Meisner, A. E. Feiguin, and E. Dagotto, Real-time simulations of nonequilibrium transport in the single-impurity Anderson model, Phys. Rev. B 79, 235336 (2009).

[20] F. Schwarz, I. Weymann, J. von Delft, and A. Weichselbaum, Nonequilibrium Steady-State Transport in Quantum Impurity Models: A Thermofield and Quantum Quench Approach using Matrix Product States, Phys. Rev. Lett. 121, 137702 (2018).

[21] A. Branschädel, G. Schneider, and P. Schmitteckert, Conductance of inhomogeneous systems: Real-time dynamics, Ann. Phys. (Berlin) 522, 657 (2010).

[22] See Supplemental Material at http://link.aps.org/supplemental/ 10.1103/PhysRevResearch.1.022006 for details.

[23] A.-P. Jauho, N. S. Wingreen, and Y. Meir, Time-dependent transport in mesoscopic systems: General formalism and applications, Semicond. Sci. Technol. 9, 926 (1994).

[24] R. Tuovinen, R. van Leeuwen, E. Perfetto, and G. Stefanucci, Time-dependent Landauer-Büttiker formula for transient dynamics, J. Phys.: Conf. Ser. 427, 012014 (2013).

[25] A. Rosch, J. Paaske, J. Kroha, and P. Wölfle, Nonequilibrium Transport through a Kondo Dot in a Magnetic Field: Perturbation Theory and Poor Man's Scaling, Phys. Rev. Lett. 90, 076804 (2003).

[26] H. Schoeller and F. Reininghaus, Real-time renormalization group in frequency space: A two-loop analysis of the nonequilibrium Kondo model at finite magnetic field, Phys. Rev. B 80, 045117 (2009); 80, 209901(E) (2009).

[27] G. Sedghi, V. M. García-Suárez, L. J. Esdaile, H. L. Anderson, C. J. Lambert, S. Martín, D. Bethell, S. J. Higgins, M. Elliott, N. Bennett, J. E. Macdonald, and R. J. Nichols, Long-range electron tunneling in oligo-porphyrin molecular wires, Nat. Nanotechnol. 6, 517 (2011).

[28] S. Schmaus, A. Bagrets, Y. Nahas, T. K. Yamada, A. Bork, M. Bowen, E. Beaurepaire, F. Evers, and W. Wulfhekel, Giant magnetoresistance through a single molecule, Nat. Nanotechnol. 6, 185 (2011).

[29] A. Bagrets, S. Schmaus, A. Jaafar, D. Kramczynski, T. K. Yamada, M. Alouani, W. Wulfhekel, and F. Evers, Single molecule magnetoresistance with combined antiferromagnetic and ferromagnetic electrodes, Nano Lett. 12, 5131 (2012).

[30] C. W. Marquardt, S. Grunder, A. Błaszczyk, S. Dehm, F. Hennrich, H. von Löhneysen, M. Mayor, and R. Krupke, Electroluminescence from a single nanotube-molecule-nanotube junction, Nat. Nanotechnol. 5, 863 (2010).

[31] G. Reecht, F. Scheurer, V. Speisser, Y. J. Dappe, F. Mathevet, and G. Schull, Electroluminescence of a Polythiophene Molecular Wire Suspended between a Metallic Surface and the Tip of a Scanning Tunneling Microscope, Phys. Rev. Lett. 112, 047403 (2014).

[32] U. Mukhopadhyay, J. P. Dehollain, C. Reichl, W. Wegscheider, and L. M. K. Vandersypen, A $2 \times 2$ quantum dot array with controllable inter-dot tunnel couplings, Appl. Phys. Lett. 112, 183505 (2018). 